\title{
The Experimental Verification of Gaussian Beam Coupling for ECH Transmission Line at $400 \mathrm{GHz}$
}

\author{
Mun Seok Choe ${ }^{1, a}$, Ashwini Sawant ${ }^{2}$ and EumMi Choi ${ }^{1,2}$ \\ ${ }^{1}$ Physics and Applied Mathematics, 44919 Ulsan National Institute of Science and Technology (UNIST), Republic of Korea \\ ${ }^{2}$ Department of Electrical and Computer Engineering, 44919 Ulsan National Institute of Science and Technology (UNIST), Republic of Korea
}

\begin{abstract}
We design a quasi-optical transmission line system for a $400 \mathrm{GHz}$ gyrotron beam. The $400 \mathrm{GHz}$ Gaussian beam is injected to a corrugated waveguide bounced from a quasi-optical mirror. From detailed $2 D$ field patterns of the output beam emitted from the corrugated waveguide, we analyze the mode contents and the source of non-ideal beam expansion
\end{abstract}

\section{INTRODUCTION}

In sub-terahertz (THz) range, development of high power transmission lines is faced with problems such as manufacturing tolerance and coupling efficiency. For preparation of KDEMO, a proceeding research is required to transfer $\mathrm{ECH}$ beam to tokamak with low loss at sub-THz frequency. A typical case is a Gaussian beam coupling from a gyrotron ouput to a corrugated waveguide. A HE11 mode in the corrugated waveguide can reduce an Ohmic loss, and the coupling of Gaussian beam coming from gyrotron to the HE11 mode is important [1]. In this research, we show the gyrotron output Gaussian beam coupling through a quasi-optical transmission line and measurement of 2D field patterns coming out the corrugated waveguide.

\section{THE COUPLING OF GAUSSIAN BEAM AND HE11 MODE}

The quasi-optical transmission line is fabricated as shown figure 1. The system consists of a quasi-optical ellipsoidal mirror, a corrugated waveguide, and a $400 \mathrm{GHz}$ ultra Gaussian beam antenna from Thomas Keating Ltd. Based on the beam waist location of the Gaussian beam antenna, the quasi-optical mirror and corrugated waveguide is designed by Surf3D simulation code [2].

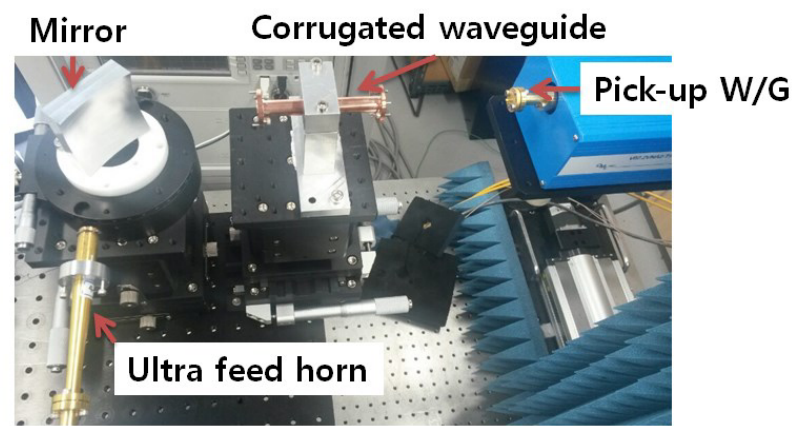

Figure 1. Experimental setup of the quasi-optical transmission line at $400 \mathrm{GHz}$

\footnotetext{
a Corresponding author: emchoi@unist.ac.kr
}

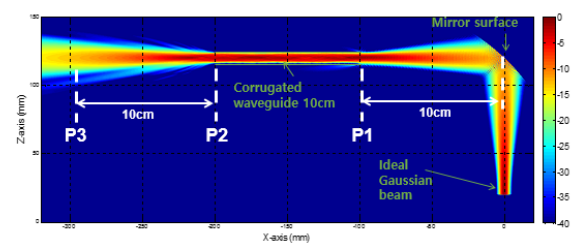

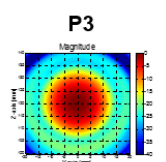

CCF : $98.86 \%$

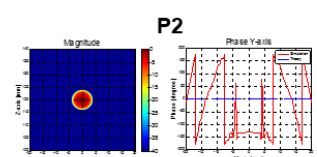

CCF : $96.49 \%$

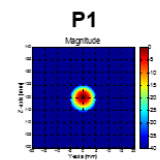

CCF : $99.81 \%$
Figure 2. Surf3D simulation results ( $P 1$ : input of corrugated waveguide, $P 2$ : output of corrugated waveguide, $P 3: 10 \mathrm{~cm}$ distance from output )

Figure 2 shows results of Surf3D simulation. The beam waist from the aperture of the Gaussian beam antenna is $2.73 \mathrm{~mm}$ and the output beam waist reflected by a mirror is also $2.73 \mathrm{~mm}$ for coupling with HE11 mode in the corrugated waveguide. Compared to theoretical results, gaussian beam is coupled with corrugated waveguide in high efficiency. In experimental setup, a precise alignment is dependent on accuracy of input beam. Thus, we measured the field pattern from ultra feed horn antenna by using a Vector Network Analyzer (N5247A) as the open waveguide is automatically adjusted by a motion controller. As illustrated in Figure 3, the gaussian beam propagation is followed to theroretical graph. Specially, the field pattern in mirror reflectance region $(10 \mathrm{~cm}$ distance from the ultra feed horn ) is well matched.
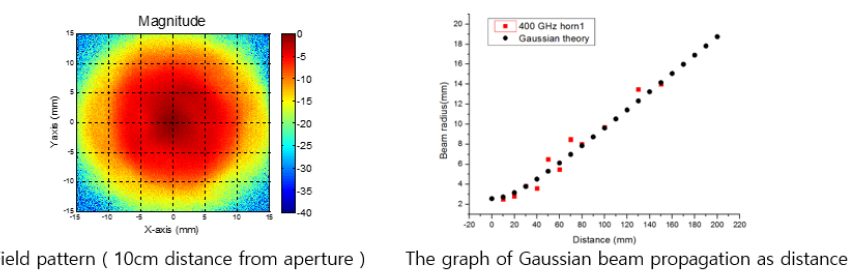

Figure 3. Gaussian beam propagation from ultra-feed horn antenna

Figure 4 shows field pattern of beam waist for coupling with the corrugated waveguide. The magnitude of electric 
field is a little bit distorted but the phase is almost flat. Based on the results, corrugated waveguide is set up to beam waist position.

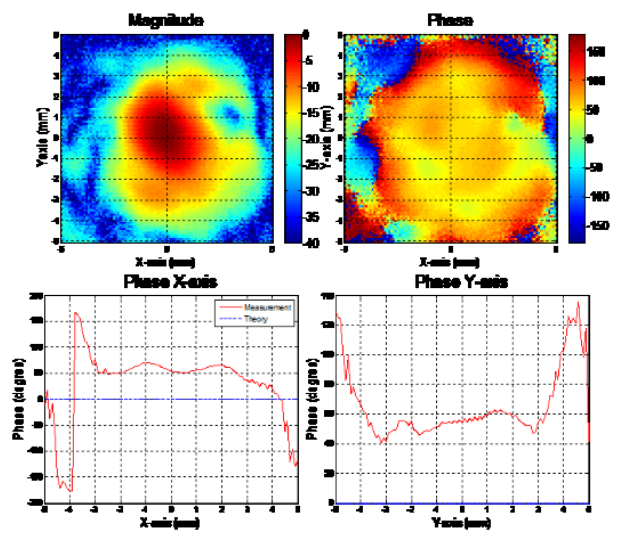

Figure 4. The beam patterns at $10 \mathrm{~cm}$ from an ultrafeed horn antenna (Beam waist position, an entrance of corrugated waveguide)

Figure 5 presents the propagated beam pattern at $10 \mathrm{~cm}$ distance from the aperture of corrugated waveguide. When compared with simulation results, the experiment data shows that the measured output beam agrees well with the Gaussian distribution although some distortion of the field pattern has been observed. A cross correlation function (CCF) is analyzed to be around $89 \%$. Considering a few $\mu \mathrm{W}$ levels and the calibration error $( \pm 0.1 \mathrm{~dB})$, some discrepancy between the measured data and ideal Gaussian case can be analyzed in sub-terahertz regions.

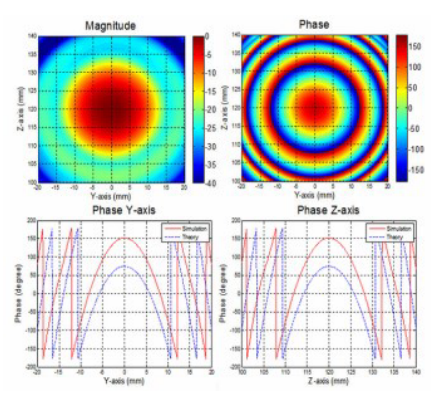

(a)

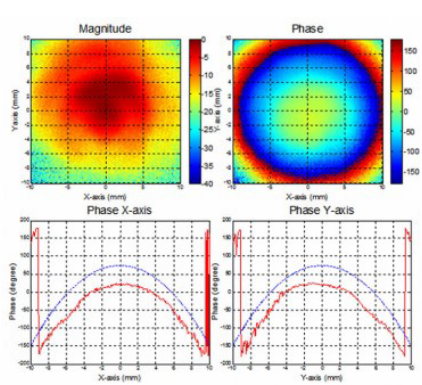

(b)
Figure 5. The beam patterns at $10 \mathrm{~cm}$ from an aperture of the corrugated waveguide (a) Surf3D simulation results (b) Experimental results

\section{CONCLUSION}

The study of the coupling with Gaussian beam and HE11 mode is performed in sub-terahertz transmission line. The experimental data have been compared with simulation results and confirmed a reasonable agreement. Futhermore, we can identify $\mathrm{THz}$ field measurement system using open ended waveguide and VNA. Through the phase correcting mirror, the system will be upgrade for high efficiency. Finally, we will proved the possibility of $\mathrm{THz} \mathrm{ECH}$ transmission line in high power. These results may help to develop advanced technology for KDEMO project.

\section{References}

1. J Kowalski, Elizabeth J. et al. "Linearly Polarized Modes of a Corrugated Metallic Waveguide." IEEE Transactions on Microwave Theory and Techniques, Vol.58, NO. 11, pp 2772-2780, 2010.

2. J. M. Neilson, and R. Bunger, "Surface integral equation analysis of quasi-optical launcher," IEEE Transactions on Plasma Science, Vol. 30, pp 794, 2002.

3. Mun Seok Choe et al. "A Comprehensive Analysis of a TE11 to HE11 Mode Converter for an Oversized Fband Corrugated Waveguide“, Journal of Electromagnetic Waves and Applications, Vol 27, Issue 17, pp 2221-2238, 2013. 